\title{
Chronic solitary plaque of the scalp: what's your diagnosis?
}

\section{Safa Idoudi', Yosra Soua', Nouha Ben Abdeljelil'², Jameleddine Zili'}

${ }^{1}$ Department of Dermatology, Fattouma Bourguiba University Hospital, Monastir, Tunisia, ${ }^{2}$ Departement of Anapathology, Fattouma Bourguiba University hospital, Monastir, Tunisia

Corresponding author: Dr. Safa Idoudi, E-mail: safaa.idoudi@gmail.com

\section{CASE REPORT}

A 60-year-old man with a history of type 2 diabetes mellitus and dyslipidemia consulted for a scalp lesion evolving for a year. The examination found an erythematous plaque of the vertex surrounded by telangiectasia and associated with perifollicular hyperkeratosis and tufted hair, without alopecia (Fig. 1). Previous local infections treated by oral antibiotherapy was reported.

Dermoscopic examination revealed decreased of follicular ostia with perifollicular hyperkeratosis and telangiectasia (Fig. 2).

Besides, the rest of the somatic examination was without abnormalities.

\section{HISTOPATHOLOGY}

Histopathological examination (hematoxylin and eosin stain) showed at higher magnification $\left({ }^{*} 100\right)$ a lymphocytic lichenoid infitrat including the hair follicule with Civatte bodies (Fig. 3). The overlying epidermis was normal.

\section{WHAT'S YOUR DIAGNOSIS?}

Answer: Lichen planopilaris (LPP).

\section{DISCUSSION}

Lichen planopilaris is an uncommon cutaneous disorder characterized by chronic lymphocytic inflammation. It is classically presenting as follicular keratotic plugs and/or perifollicular scaling with perifollicular erythema

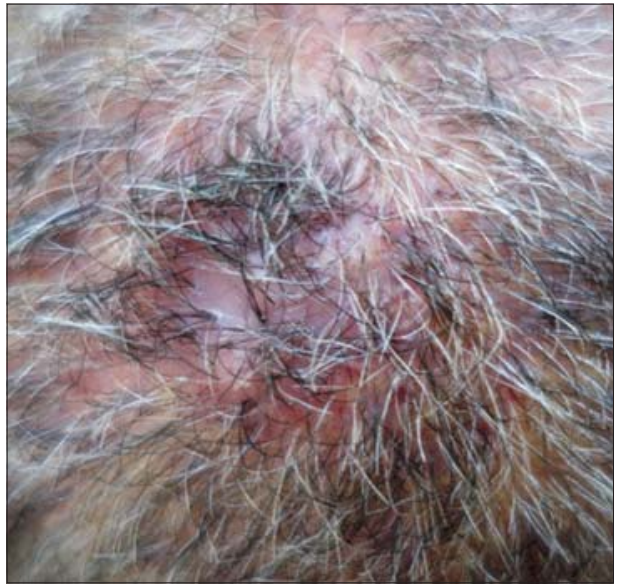

Figure 1: Erythematous plaque of the vertex surrounded by telangiectasia and associated with perifollicular hyperkeratosis and tufted hair, without alopecia.

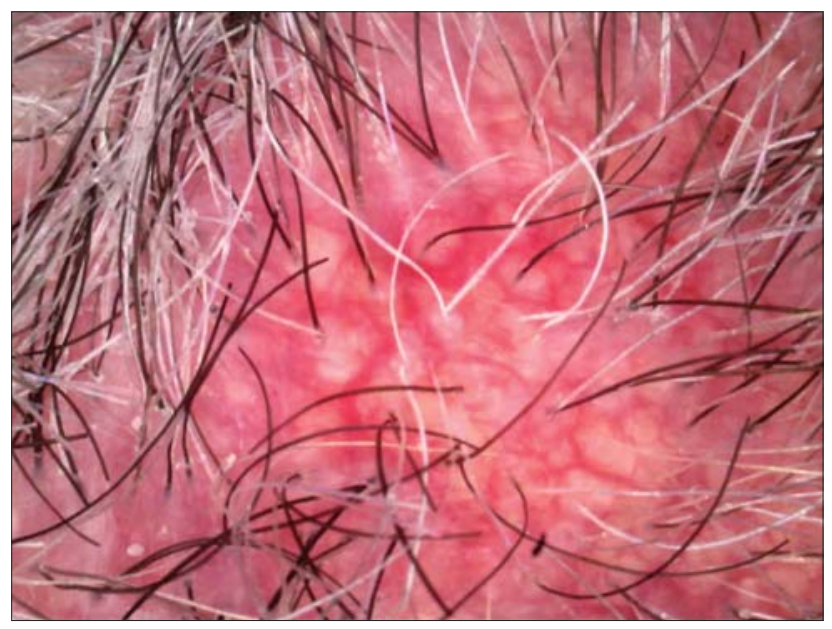

Figure 2: Dermoscopic photograph showing a decrease in the number of follicular ostia with perifollicular hyperkeratosis and telangiectasia ( ${ }^{5} 50$, Dino-lite pro-polariser USB dermoscope).

leading to scarring alopecia [1]. The absence of alopecia is uncommon and could be seen at an early stage of the

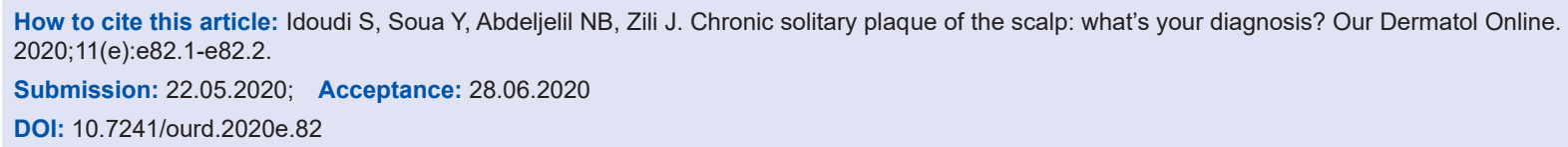




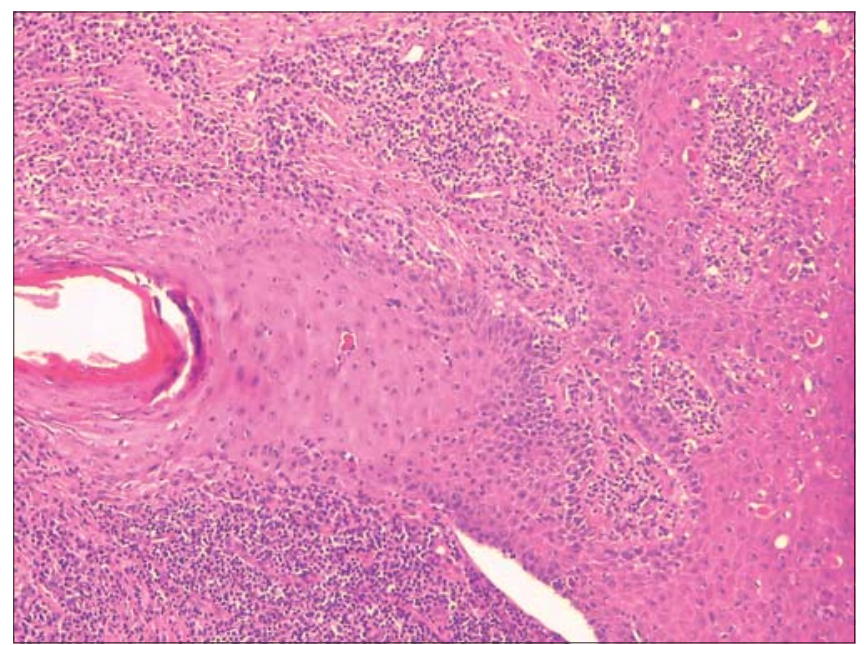

Figure 3: Histological aspect showing a lymphocytic infiltrate involving the isthmus and infundibulum of the hair follicle with Civatte bodies $\left(\mathrm{HE}^{*} 100\right)$.

disease. Dermoscopy can contribute to the diagnosis of LPP and even assess its activity by showing in the center of the bald areas a lack of follicular orifices and on the margin, a pink perifollicular inflammation with keratin scale surrounding and extending along the proximal part of the hair shaft [2]. These findings histologically correspond to perifollicular hyperkeratosis and vacuolar changes with the presence of necrotic keratinocytes in the basal layer of the hair follicle [3]. Other aspects may be found such as white dots or patches, pigment network or blue-grey pattern around white dots and follicles realizing a target pattern [4].

LPP is associated with cutaneous or mucosal lesions of lichen planus in about $30 \%$ of cases [5]. Management often involves topical or intralesional steroids as a first-line treatment in limited forms otherwise hydroxychloroquine, acitretin and mycophenolate mofetil can be used. As cicatricial alopecia, the main goal of therapy is to prevent the progression of the lesions.

\section{Consent}

The examination of the patient was conducted according to the Declaration of Helsinki principles.

The authors certify that they have obtained all appropriate patient consent forms. In the form the patient(s) has/have given his/her/ their consent for his/her/their images and other clinical information to be reported in the journal. The patients understand that their names and initials will not be published and due efforts will be made to conceal their identity, but anonymity cannot be guaranteed.

\section{REFERENCES}

1. Assouly P, Reygagne P. Lichen planopilaris: uptade on diagnosis and treatment. Semin Cutan Med Surg. 2009;28:3-10.

2. Lajevardi V, Mahmoudi H, Moghanlou S, Ansari M, Teimourpour A, Daneshpazhooh M. Assessing the correlation between trichoscopic features in lichen planopilaris and lichen planopilaris activity index. Australas J Dermatol. 2019;60:214-8.

3. Soares VC, Mulinari-Brenner F, Souza TE. Lichen planopilaris epidemiology: a retrospective study of 80 cases. An Bras Dermatol. 2015;90:666-70.

4. Rakowska A, Slowinska M, Kowalska-Oledzka E, Warszawik O, Czuwara J, Olszewska M, et al. Trichoscopy of cicatricial alopecia. J Drugs Dermatol. 2012;11:753-8.

5. Weston G, Payette M. Update on lichen planus and its clinical variants. Int J Womens Dermatol. 2015;1:140-9.

Copyright by Safa Idoudi, et al. This is an open access article distributed under the terms of the Creative Commons Attribution License, which permits unrestricted use, distribution, and reproduction in any medium, provided the original author and source are credited.

Source of Support: Nil, Conflict of Interest: None declared. 